\title{
Contribuciones del Programa de Investigaciones en Frijol en Centro América y El Caribe
}

\author{
Juan Carlos Rosas ${ }^{1}$
}

Resumen. El Programa de Investigaciones en Frijol (PIF) es un programa regional enfocado en el mejoramiento genético del frijol común (Phaseolus vulgaris L.) que enfatiza en la generación y diseminación de variedades mejoradas de las clases comerciales de frijol de grano rojo y negro. Su enfoque regional abarca los países de Centro América y el Caribe dónde se cultivan estos dos tipos de granos. Sus clientes principales son los pequeños productores de frijol y las organizaciones públicas y privadas involucradas en el desarrollo y transferencia de tecnologías para este cultivo básico. Los énfasis en mejoramiento genético del PIF son la resistencia a enfermedades, la tolerancia a la sequía, la baja fertilidad de los suelos y las altas temperaturas. El mejoramiento genético es realizado utilizando técnicas y métodos convencionales, mejoramiento participativo, selección asistida con marcadores, y la evaluación y validación regional a través de los programas miembros de la Red de Frijol. El PIF y sus colaboradores han liberado más de 40 variedades mejoradas de frijol ampliamente utilizadas por pequeños agricultores de Centro América y Haití, lo que representan un impacto significativo en la seguridad alimentaria y calidad de vida de las familias beneficiarias. Los colaboradores del PIF incluyen a programas nacionales, organizaciones no gubernamentales, el Centro Internacional de Agricultura Tropical, los programas Bean/Cowpea y Dry Grain Pulses CRSP, y el Programa Colaborativo de Fitomejoramiento Participativo para Mesoamérica.

Palabras clave: Altas temperaturas, baja fertilidad, Phaseolus vulgaris L., resistencia a enfermedades, sequía, variedades mejoradas.

\section{Contributions of the Bean Research Program in Central America and the Caribbean}

\begin{abstract}
The Bean Research Program (PIF, acronyms in Spanish) is a regional program focused on the genetic improvement of common beans (Phaseolus vulgaris L.), with an emphasis in the generation and dissemination of improved varieties from the small red and black bean commercial classes. Its regional focus includes the countries of Central America and the Caribbean where these two grain bean classes are grown. The clients of the PIF are mainly small bean producers and public and private organizations involved in the development and transfer of technologies of this basic crop. The emphasis on genetic improvement of this program is on disease resistance and tolerance to drought, low soil fertility and high temperatures. The genetic improvement is done by using conventional breeding techniques, participatory plant breeding, marker assisted selection and regional evaluation and validation through the program members of the Bean Regional Network. The PIF and collaborators have released more than 40 improved bean cultivars which are extensively used by small farmers in Central America and Haiti, representing a significant impact in food security and quality of life for the families of the beneficiaries. The PIF's collaborators includes national programs, non-governmental organizations, the International Center for Tropical Agriculture, the Bean/Cowpea CRSP and Dry Grain Pulses CRSP programs, and the Participatory Plant Breeding Collaborative Program for Mesoamerica.
\end{abstract}

Key words: Disease resistance, drought, high temperatures, improved varieties, low fertility, Phaseolus vulgaris L.

\section{Introducción}

El Programa de Investigaciones en Frijol (PIF) de la Escuela Agrícola Panamericana (EAP), Zamorano, Honduras, inició sus actividades en 1988. Su enfoque principal ha sido la generación de variedades mejoradas de frijol con mejor adaptación agronómica y mayor productividad que las variedades comerciales tradicionales utilizadas, como resultado de una estrategia de mejoramiento para incrementar la resistencia a enfermedades y tolerancia a factores abióticos que limitan su productividad en Centro América y el Caribe.

Los objetivos del PIF han estado dirigidos a contribuir en la seguridad alimentaria y nutricional y el mejoramiento de la calidad de vida de los pequeños

\footnotetext{
${ }^{1}$ Escuela Agrícola Panamericana, Apartado Postal 93, Tegucigalpa, Honduras, correo electrónico jcrosas@ zamorano.edu
} 
productores de frijol y sus familias. Durante el período 1988-2000, el PIF concentró sus actividades en ampliar la base genética e incrementar la resistencia a las enfermedades y la tolerancia a los factores abióticos predominantes (sequía, altas temperaturas y baja fertilidad de los suelos) utilizando recursos genéticos y el mejoramiento convencional. Posteriormente, se implementaron las técnicas moleculares para la caracterización de la diversidad genética del frijol y la selección asistida con marcadores (SAM) para la resistencia a enfermedades. En el 2000, el PIF implementó en sus actividades la metodología de fitomejoramiento participativo (FP) para desarrollar variedades mejoradas con mayor adaptación a nichos agroecológicos específicos, llevando a cabo los procesos de evaluación y selección en comunidades metas, con la participación de agricultores organizados en comités técnicos.

El aumento en rendimiento al usar variedades mejoradas desarrolladas por el PIF en colaboración con programas nacionales e internacionales, universidades de los Estados Unidos, organizaciones no gubernamentales (ONG) y grupos de agricultoresexperimentadores, ha sido potenciado de manera complementaria con el uso de semilla de calidad producida y diseminada localmente por programas nacionales y ONG. En estos esfuerzos de producción de semilla de calidad, el PIF ha jugado un papel muy importante proveyendo semilla básica (fundación) de las variedades liberadas, a los usuarios que participan en la cadena de valor de la producción de semilla de frijol en Centro América y el Caribe.

La implementación de prácticas de manejo del cultivo con un enfoque agroecológico, utilizando abonos orgánicos y productos naturales disponibles en las comunidades, ha incrementado la productividad del cultivo en zonas donde predominan las condiciones de bajo uso de insumos, característico de la mayoría de los pequeños productores de frijol en la región. Las contribuciones tecnológicas del PIF han incrementado la productividad, mejorado la seguridad alimentaria y la calidad de vida de muchos pequeños productores y sus familias. El desarrollo de capacidades de técnicos y agricultores líderes a través de cursos y talleres, entrenamiento en servicio y aprender haciendo, han sido algunas de las contribuciones al desarrollo de talento en la región de influencia del PIF.

\section{El Cultivo de Frijol y sus Limitantes}

El frijol común (Phaseolus vulgaris L.) es una fuente de proteínas y calorías importante y un alimento esencial en la dieta diaria de miles de personas en las zonas rurales y en las áreas urbanas de menor ingreso de Centroamerica (Rosas 2000; Rosas et al. 2000a; Rosas et al. 2000b). El frijol es cultivado mayormente por pequeños agricultores en fincas de menos de 3.0 ha ubicadas en zonas de laderas y suelos marginales, y bajo una agricultura de bajo uso de insumos y tecnologías por falta de recursos y acceso limitado (Rosas 2000; Rosas et al. 2000a).

El cultivo de frijol es afectado principalmente por enfermedades virales causadas por el virus del mosaico común (VMCF) y el virus del mosaico dorado amarillo (VMDAF), la bacteriosis (Xanthomonas campestris pv. phaseoli), la antracnosis (Colletotrichum lindemuthianum), la mancha angular (Phaeoisariopsis griseola), la mustia hilachosa (Thanatephorus cucumeris) y la roya (Uromyces appendiculatus). Además, el cultivo es afectado en la región del Caribe por la cepa necrótica del virus del mosaico común del frijol (VMCNF).

Las plagas más importantes que afectan al cultivo del frijol son las que atacan a las plántulas incluyendo los gusanos cortadores (Agrotis spp., Feltia spp., Spodoptera spp.), el barrenador menor del tallo (Elasmopalpus lignosellus) y la gallina ciega (Phyllophaga spp.); las plagas defoliantes incluyendo a los crisomélidos (Diabrotica spp. y Cerotoma spp.), el gusano medidor (Trichoplusia ni), el falso medidor (Pseudoplusia includens), el gusano cabezón (Urbanus proteus), el gusano peludo (Estigmene acrea), el pegahojas o telarañero (Hedylepta indicata), los gusanos soldados o militares (Spodoptera spp.) y la babosa (Sarasinula plebeia). Los insectos chupadores incluyen el saltahojas o lorito verde (Empoasca spp.), pulgones o áfidos (Aphis spp.) y mosca blanca (Bemisia tabaci); las plagas de las vainas incluyendo el picudo (Trichapion godmani) y perforadores de vainas (Epinotia aporema, Helicoverpa zea); y los gorgojos de almacén (Zabrotes subfasciatus y Acanthoscelides obtectus).

El estrés hídrico causado por la sequía afecta al cultivo debido, principalmente, a que es sembrado después del maíz. Más del $60 \%$ del frijol en Centro 
América es sembrado en la época de postrera en la etapa final de la temporada de lluvias y en la que frecuentemente los cultivos son afectados por sequía terminal (Frahm et al. 2003; Frahm et al. 2004). En las regiones más húmedas, el frijol es sembrado bajo humedad residual en la época conocida como apante o postrera tardía, al final de la temporada de lluvias. Bajo estas condiciones se pueden presentar condiciones extremas de alta humedad hasta la madurez de cosecha o estrés de sequía por precipitaciones menores a las esperadas.

La productividad del frijol es reducida significativamente por la baja fertilidad de los suelos que predominan en las zonas donde se cultiva en América Latina y en África (Graham et al. 2003). Las deficiencias causadas por el bajo contenido de materia orgánica, y el nitrógeno derivado de su descomposición, fósforo, calcio, magnesio y otros nutrimentos caracterizan a la mayoría de los suelos donde se cultiva frijol en Centro América (Rosas 2001; Graham et al. 2003). La falta de recursos de los pequeños agricultores limita el uso de prácticas de conservación y manejo que podrían resultar en mejores condiciones de los suelos y mayor productividad del frijol.

En algunas regiones se presenta la posibilidad de producir frijol en zonas costeras con suelos más adecuados al cultivo y el uso de sistemas de riego, sobretodo en rotación con cultivos de exportación. En estas zonas bajas, las temperaturas son más altas que en las zonas tradicionales, limitando la utilización de estas condiciones de suelos y disponibilidad de riego más favorables para el cultivo de frijol. Además, la tendencia al aumento de la temperatura por el cambio climático en las zonas tradicionales de producción de frijol, plantean la necesidad de tener variedades con mayor tolerancia al calor, que las usadas tradicionalmente (Porch et al. 2007).

\section{Mejoramiento de la Resistencia a Enfermedades y Tolerancia a Factores Abióticos}

Resistencia a enfermedades. La generación de variedades mejoradas resistentes a enfermedades ha sido el enfoque principal del mejoramiento genético en el PIF. El mejoramiento de la resistencia a enfermedades se enfocó en la transferencia de genes específicos a variedades y líneas élites y la ampliación de la base genética utilizando fuentes de germoplasma provenientes de los reservorios genéticos andino y mesoamericano. Desde el inicio, el mejoramiento se enfocó hacia la resistencia múltiple partiendo como base el desarrollo de variedades mejoradas resistentes al VMCF y al VMDAF en combinación con resistencias a por lo menos una enfermedad adicional (Blair et al. 1994; Rosas et al. 2000a; Rosas et al. 2000b; Beaver et al. 2003). La resistencia al VMCF ha estado fundamentada en la incorporación del gen dominante $I$ y todo material mejorado que se distribuye para evaluación a nivel regional a través de la Red de Frijol debe contener este gen. Sin embargo, la presencia de la cepa necrótica de este virus (el VMCNF) en la región del Caribe, ha conducido a una estrategia para incorporar el gen recesivo $b c 3$, para proteger al gen $I$ en presencia de esta cepa necrótica. Este enfoque se ha ampliado para Centro América ya que con los incrementos de temperatura por efectos del cambio climático, el cultivo en la región se presenta más vulnerable a ser afectado por el VMCNF.

Las enfermedades a las cuales se les ha prestado atención en el desarrollo de variedades con resistencia múltiple, además de las causadas por el VCMF y el VMDAF, son la antracnosis, bacteriosis común, mancha angular, mustia hilachosa y roya (Serracín et al. 1991; Rosas et al. 2000a; Steadman et al. 2002; Beaver et al. 2003; Acevedo et al. 2005; Gonzalez et al. 2005; Acevedo et al. 2008; Rodriguez y Rosas 2010; Acevedo et al. 2012). Desde 1996 se han distribuido a los países miembros de la Red de Frijol (Costa Rica, El Salvador, Haití, Honduras, Guatemala, Nicaragua, Panamá, República Dominicana y Puerto Rico), los Viveros de Adaptación Centroamericano (VIDAC) y Ensayos Centroamericano de Adaptación y Rendimiento (ECAR), que contienen líneas mejoradas de grano rojo y negro con resistencia múltiple (Rosas et al. 2000a; Beaver et al. 2003). Para lograr estos avances en el mejoramiento genético de la resistencia a enfermedades, se han evaluado germoplasmas para seleccionar progenitores, familias y líneas resistentes, empleando técnicas de evaluación de la incidencia y severidad de estas enfermedades y teniendo en cuenta la diversidad patogénica de sus agentes causales (Blair et al. 1994; Rosas et al. 2000a; 
Rosas et al. 2000b; Beaver et al. 2002; Steadman et al. 2002; Beaver et al. 2003; Beaver et al. 2008; Gonzalez et al. 2005; Acevedo et al. 2008; Rodriguez y Rosas 2010; Acevedo et al. 2012).

Tolerancia a altas temperaturas. La posibilidad de sembrar frijol en regiones bajas con suelos planos más fértiles y mayor acceso a sistemas de irrigación, representa condiciones para obtener rendimientos superiores al promedio. En muchas de estas zonas potenciales de Centro América se producen cultivos de exportación incluyendo hortalizas y frutales utilizando alta tecnología; donde se requieren cultivos de rotación de buen valor comercial como frijol. En estas zonas, las temperaturas son frecuentemente más altas a las de las zonas tradicionales de producción de frijol, por lo que la mayoría de las variedades tradicionales no logran adaptarse. La utilización de variedades mejoradas de frijol tolerantes al calor es necesaria para garantizar la producción de frijol en estas zonas. Con los incrementos en temperatura por efectos de cambio climático, el mejoramiento para tolerancia a altas temperaturas aún en zonas tradicionales es un componente importante en la estrategia de desarrollo de variedades mejoradas para Centro América y el Caribe (Porch et al. 2007).

El PIF y sus colaboradores han identificado accesiones tolerantes al calor con adaptación a estas condiciones para generar variedades comerciales que combinen la resistencia a enfermedades con la tolerancia al estrés causado por las altas temperaturas (Rosas et al. 2000b; Porch et al. 2007). Algunas de las variedades generadas en los últimos años tienen mejor adaptación al calor que las tradicionales, siendo la variedad de grano rojo CENTA Pipil, liberada en El Salvador en el 2005, una de las más tolerantes a este estrés. El PIF colabora con científicos de la Universidad de Puerto Rico (UPR) y la Estación Experimental de Agricultura Tropical del Departamento de Agricultura de los Estados Unidos (TARS/USDA, por sus siglas en inglés) en el desarrollo de variedades de frijol tolerantes al calor.

Tolerancia a sequía y baja fertilidad. El estrés hídrico causado por la sequía y la baja fertilidad de los suelos son los factores abióticos más importantes y afectan más del $70 \%$ de las regiones productoras de frijol en el mundo; con frecuencia, estas limitantes ocurren juntos en el trópico (Graham et al. 2003; Frahm et al. 2003; Ho et al. 2005; Henry et al. 2010). Debido a que los pequeños productores de frijol en Centro América y Haití no poseen los recursos que les permitan modificar estas limitaciones, empleando prácticas de conservación y manejo de suelos y el uso de sistemas de irrigación, la sequía y la baja fertilidad, se reduce la productividad de sus cultivos. Por lo tanto, el PIF y sus colaboradores de la UPR y Pennsylvania State University (PSU), han investigado y desarrollado variedades tolerantes a estos estreses abióticos.

La estrategia de mejoramiento para tolerancia a la sequía y la baja fertilidad consiste en la identificación de genotipos con comportamiento agronómico superior bajo condiciones de estrés. La mayor eficiencia en el crecimiento y productividad de las variedades mejoradas bajo estas limitantes, se traduce en mayor vigor vegetativo y rendimiento de grano que las variedades tradicionales. Los estudios indican la importancia de la arquitectura de las raíces, es decir la configuración espacial del sistema radical, en determinar la adquisición de recursos debajo de la superficie (Ho et al. 2005; Lynch 2005). La importancia de la arquitectura de raíces en la productividad de la planta, radica en que la distribución espacial del sistema radical determina la habilidad de la planta para explotar los nutrimentos y agua disponibles en el suelo (Ho et al. 2005; Lynch 2005; Henry et al. 2010). La tolerancia a la sequía en el frijol ha sido asociada a mayor profundidad de raíces; mientras que, la mayor adquisición de nutrimentos está asociada a mayor exploración de la capa arable, sobretodo en el caso de elementos inmóviles como el fósforo. Debido a esto, la variación genética de la arquitectura de las raíces está asociada a la adaptación y productividad en ambientes específicos (Lynch 2005).

Antes, la evaluación de germoplasma de frijol y el mejoramiento de la tolerancia a la sequía (Rosas et al. 1991; Frahm et al. 2003) y la baja fertilidad (Rosas et al. 1996), se enfocaban independientemente. Con ambas limitantes en la mayoría de las zonas productoras de frijol en Centro América y el Caribe, el desarrollo de variedades tolerantes a ambos factores se maneja de manera integrada con la recombinación 
genética aportada por progenitores tolerantes; como resultado de este enfoque, las variedades mejoradas liberadas en los últimos años son más tolerantes a ambos estreses que las variedades tradicionales. Una alternativa adicional es el desarrollo de variedades multilíneas compuestas por líneas endogámicas que poseen diferente arquitectura de raíces que le permite a la variedad mejor respuesta a las condiciones variables de humedad y fertilidad del suelo (Henry et al. 2010). La generación de multilíneas requiere el uso de métodos de mejoramiento y selección que permiten desarrollar líneas endogámicas con antecedente genético similar, lo que permite que la variedad pueda estar conformada por líneas agronómicamente (días a madurez, hábito de crecimiento) y comercialmente (tamaño, forma y color del grano) uniformes, pero que difieran en la arquitectura de raíces para una mejor adaptación a condiciones variables de nutrimentos y humedad del suelo (Ho et al. 2005; Henry et al. 2010).

\section{Fijación Biológica de Nitrógeno}

La producción de frijol en América Latina y África está más concentrada en fincas pequeñas, donde predominan los suelos de baja fertilidad, principalmente con deficiencias de nitrógeno y fósforo (Graham et al. 2003). Una de las alternativas más viables para aumentar la productividad en suelos bajos en nitrógeno es a través del mejoramiento genético de la capacidad de fijación biológica de nitrógeno (FBN) (McFerson et al. 1982; Rosas 1986a; Rosas 1986b; Rosas y Bliss 1986; Rosas et al. 1987). La amplia diversidad genética en la capacidad de nodulación y FBN del frijol común (Graham y Rosas 1977; Graham y Rosas 1978a; Graham y Rosas 1978b; Graham et al. 2003), hace factible el desarrollo de variedades mejoradas con mejor adaptación a suelos pobres en nitrógeno. El PIF utiliza este enfoque de mejoramiento de la FBN en el desarrollo de variedades mejoradas para Centro América y el Caribe.

\section{Fitomejoramiento Participativo}

En 1999, el PIF incorporó el enfoque de fitomejoramiento participativo (FP) en sus actividades de mejoramiento genético en la región centroamericana. Con este enfoque se facilitó la integración de pequeños productores organizados en comités de investigación, en el desarrollo de variedades mejoradas mejor adaptadas a los sistemas de producción de las comunidades y una mayor adopción de estas tecnologías por los agricultores (Rosas 2001; Rosas et al. 2003; Rosas et al. 2009a; Hocdé et al. 2010).

Como parte del Programa Colaborativo para el Fitomejoramiento Participativo en la Región de Mesoamérica (FP-MA), desde el 2000, el PIF participa en la generación y diseminación de variedades mejoradas usando metodologías de FP en la región de Centro América. Como resultado de los esfuerzos en $\mathrm{FP}$, muchas variedades han sido desarrolladas bajo este enfoque y se han convertido en las variedades preferidas en las localidades donde fueron generadas con participación de los productores (Rosas et al. 2003; Araya y Hernández 2007; Rosas et al. 2009a; Hocdé et al. 2010). En Honduras, 18 variedades de frijol rojo han sido desarrolladas en colaboración con miembros de los Comités de Investigación Agrícola Local (CIAL) de más de 50 comunidades rurales de la región del Lago Yojoa y los departamentos de Yoro y Francisco Morazán (Cuadro 1). En Costa Rica, las variedades liberadas desde el 2000 han sido desarrolladas a través de la metodología FP (Hernández y Elizondo 2006; Araya y Hernández 2007). En Nicaragua, se han generado tres variedades de frijol rojo a través de este enfoque (Rosas et al. 2009a; Hocdé et al. 2010). Recientemente, en El Salvador se liberaron tres variedades de frijol de grano rojo desarrolladas mediante esta metodología participativa.

\section{Los Recursos Genéticos del Frijol}

Las actividades de mejoramiento del PIF se han conducido basado en la diversidad genética del cultivo provenientes de colecciones de germoplasma de Honduras, la cual contiene más de 600 accesiones incluyendo variedades criollas o tradicionales de frijol común y parientes del género Phaseolus ( $P$. coccineus, $P$. lunatus y $P$. acutifolius) colectadas en la década de los 90 y conservadas en el Banco de Germoplasma de Zamorano. Este germoplasma fue ampliado con colecciones de $P$. vulgaris silvestres durante 2000-04 (Acevedo et al. 2005; Acevedo et al. 2010). Las 
accesiones de frijol común de la colección hondureña han sido caracterizadas usando descriptores morfológicos (Rosas et al. 2009b; Meza et al. 2012), marcadores moleculares (Guachambala y Rosas 2010) y utilizadas en estudios de la diversidad patogénica del agente causal de la roya del frijol (Acevedo et al. 2008; Acevedo et al. 2010).

Cuadro 1. Variedades desarrolladas por el Programa de Investigaciones en Frijol (PIF) mediante la aplicación de la metodología de Fitomejoramiento Participativo en Centro América.

\begin{tabular}{lll}
\hline Variedades & País (colaboradores) \\
\hline Amilcar, Briyo AM, & Honduras (FIPAH, PRR, \\
Campechano JR, Cedrón, & CIAT, INTA, \\
Chepe, Conán 33, Don & ASOCIALAYO, ASOCIAL \\
Cristóbal, Don Kike, & Yorito, ASOCIAL \\
Esperanceño, Macuzalito, & Vallecillo) \\
La Majada AF, Marcelino, & \\
Milagrito, Nueva Esperanza & \\
01, Paisano FP, Palmichal 1, \\
San José y Victoria \\
Bribrí, Cabecar, Telire, \\
Curré, Gibre, Surú, Tayní y \\
Tonjibe, \\
Luisito, Marrojo y Río Rojo & Nicaragua (CIPRES, INTA) \\
Guazapa 1, La Presa y San & El Salvador (CENTA) \\
Antonio FP1 & \\
\hline
\end{tabular}

Muchas accesiones utilizadas como fuentes de resistencia a enfermedades fueron obtenidas del CIAT a través de diversos viveros, como VIFURE y VIPADOGEN, distribuidos por esta institución en la década de los 90. Las fuentes de tolerancia a sequía y baja fertilidad utilizadas en los últimos años fueron obtenidas a través de la colaboración con el CIAT bajo los proyectos de Agrosalud y Red SICTA. De la misma manera, el PIF ha obtenido accesiones de germoplasma y líneas mejoradas a través de la colaboración con la Universidad de Puerto Rico y Universidad de Nebraska, bajo los programas del Bean/Cowpea y Dry Grain Pulses CRSP.

\section{Selección Asistida con Marcadores}

Desde el 2002, el PIF utiliza marcadores moleculares SCAR (región amplificada de secuencia caracterizada) en la selección asistida con marcadores (SAM), para complementar las selecciones en el campo e invernadero basado en la incidencia y severidad de las reacciones a las principales enfermedades del cultivo de frijol. La utilización de la SAM se concentra en la depuración de las variedades anteriormente liberadas y la selección de líneas promisorias en procesos de validación, previa a la liberación en los países de CA/C. En la actualidad, se caracterizan en el Laboratorio de Biotecnología Aplicada de Zamorano las variedades liberadas y líneas promisorias con los siguientes marcadores SCAR ligados a la resistencia a enfermedades del frijol común: SR2 (gen bgml) y SW12 (QTL mayor) de resistencia al VMDAF; SW13 (gen I) de resistencia al VMCF; SAP 6 y SU91 (QTL mayores) de resistencia a la bacteriosis común; SH13 (gen Phgl) y SNO2 (gen Phg2) de resistencia a la mancha angular; SK14 (gen Ur3), SA14 (gen Ur4), SI19 (gen $U r 5$ ), SBC6 (gen $U r 6$ ), SAD12 (gen $U r 7$ ), UR11/GT2 (gen $\mathrm{Ur} 11$ ) de resistencia a la roya; y SAS13 (gen $\mathrm{Co}$ $4^{2}$ ), SCAreoli (gen Co-2) y SAB3 (gen Co-5) de resistencia a la antracnosis.

\section{Contribuciones e Impactos del Programa de Investigaciones en Frijol}

El PIF se organizó en 1988 para integrar acciones de proyectos y solucionar problemas específicos del cultivo de frijol, a través de una estrategia propia de programa de mejoramiento genético con visión y acciones integradas para alcanzar objetivos a largo plazo. Mediante esta estrategia, el PIF desarrolla las condiciones para facilitar la continuidad necesaria que permitiera contribuir a aumentar la productividad del cultivo de frijol y el bienestar de las familias de los pequeños productores que dependen de este cultivo para su seguridad alimentaria y nutricional y la generación de ingresos, a través de variedades mejoradas con comportamiento agronómico superior a las tradicionales. 
Desde su inicio, el enfoque principal del PIF ha sido el desarrollo y generación de variedades mejoradas de frijol común, teniendo en cuenta la problemática del cultivo a nivel regional descrita anteriormente. Las primeras variedades de frijol de grano rojo pequeño desarrolladas por el PIF, fueron principalmente el resultado de mayor resistencia a enfermedades, principalmente al VMCF y al VMDAF, mejor adaptación agronómica y buena aceptación comercial y de consumo, lo que permitió su diseminación regional en varios países de Centro América y Haití, como es el caso de las variedades Tío Canela 75 y Amadeus 77 (Rosas et al. 1997; Rosas et al. 2004a; Rosas et al. 2004b). Posteriormente, se desarrollaron variedades resistentes a enfermedades con mejor adaptación a suelos de baja fertilidad como Bribrí de amplia diseminación en Costa Rica (Rosas et al. 2003; Hernández y Elizondo 2006), y la variedad de grano negro Aifi Wuriti en Haití.

Ante el incremento de la demanda de frijol de grano rojo claro (más generalizado en variedades tradicionales) por el crecimiento en los mercados regionales y de las ciudades de Estados Unidos con alta población centroamericana, el PIF enfatiza, a partir del 2000, en el desarrollo de variedades mejoradas tipo Rojo de Seda (nombre como se le conoce a diferentes variedades comerciales con grano tipo rojo claro en estos mercados), resistentes a enfermedades y con mejor adaptación agronómica. Las primeras variedades mejoradas tipo Rojo de Seda fueron liberadas a partir del 2007, incluyendo a DEORHO y Cardenal en Honduras; INTA Matagalpa, INTA Seda y recientemente INTA Centro Sur en Nicaragua; y CENTA CPC, CENTA Nahuat y CENTA Chaparrastique en El Salvador. Nuevas variedades del tipo Rojo de Seda fueron desarrolladas de variedades criollas tradicionales de Costa Rica, como Sacapobres de la que se derivaron las variedades mejoradas Gibre y Curré liberadas en el 2007.

En colaboración con los programas nacionales de Guatemala y Haití, se liberaron variedades mejoradas de grano negro, el frijol más consumido en estos países. Entre las variedades mejoradas de frijol negro liberadas en Guatemala están ICTAZAM ML, ICTA Sayaxché e ICTA Petén (colaboración con el CIAT); y en Haití las variedades Aifi Wuriti y DPC 40 (colaboración con UPR, Universidad de Nebraska y el
IDIAF de la República Dominicana). Nuevas variedades de grano negro están siendo validandas en estos países, en Nicaragua y El Salvador.

En los últimos años, en Centro América ha aumentado el rendimiento usando variedades mejoradas resistentes a enfermedades y con mejor adaptación agronómica (Beaver et al. 2003; Hernández y Elizondo 2006; Reyes et al. 2012). Sin embargo, los mayores incrementos en rendimientos es cuando se usa además semilla de calidad de estas variedades mejoradas, y el manejo integrado del cultivo emplea programas de fertilización y de manejo de plagas con insumos químicos recomendados, o cuando se usan recursos disponibles localmente bajo un enfoque agroecológico.

En el 2001, se hizo un estudio para estimar el impacto económico de la investigación en frijol en Honduras, representado principalmente por el desarrollo de variedades mejoradas de frijol resistentes a enfermedades (Mather et al. 2003). Los resultados de las encuestas en fincas de las dos principales regiones de producción de frijol en Honduras, El Paraíso y Olancho, mostraron que el $41-46 \%$ de los productores de frijol adoptaron variedades resistentes que fueron diseminadas en la década 1990-2000.

En el 2004, el 70\% del área sembrada en Costa Rica fue con tres variedades mejoradas (Bribri, Cabecar y Telire) desarrolladas por el PIF en colaboración con el PITTA-Frijol (Hernández y Elizondo 2006). El estudio indicó que, según los agricultores entrevistados de la Región Brunca, la susceptibilidad a enfermedades de las variedades tradicionales fue la razón de su remplazo por las variedades mejoradas mencionadas.

El impacto de las variedades mejoradas de frijol desarrolladas por el PIF, en colaboración con investigadores del Bean/Cowpea and Dry Grain Pulses CRSP, la UPR, el CIAT y la Red de Frijol conformada por los programas nacionales de Centro América y el Caribe, fue estudiado por Reyes et al. (2012). Los resultados indican que las variedades mejoradas en el 2010 eran el 67\% del área sembrada con frijol rojo pequeño en Centro América, y 50\% en el Norte del Ecuador, que fue incluida también en el estudio. Además, las inversiones en investigación para el desarrollo de estas variedades han generado más de 350 millones de dólares. 
Un estudio conducido por Porch et al. (2007), en la Costa Norte de Honduras, sugiere que una proporción significativa de la producción de frijol se desarrolla bajo condiciones de estrés de altas temperaturas. Las diferencias en rendimiento de frijol entre las aldeas de baja y alta altitud fueron causadas por limitaciones climáticas atribuidas a la producción en diferente elevación. Bajo dicho escenario, el análisis de costo-beneficio indicó que el desarrollo e introducción de variedades tolerantes al calor pueden resultar en ganancias sustanciales para el agricultor, mediante la disminución de los problemas asociados al aumento de las temperaturas por el cambio climático.

\section{Literatura Citada}

Acevedo, M., J.R. Steadman, J.C. Rosas y J. Venegas. 2005. Characterization of virulence diversity of the bean rust pathogen Uromyces appendiculatus in wild bean populations as a tool for effective resistance gene deployment. Annual Report of the Bean Improvement Cooperative 48:132-133.

Acevedo, M., J.R. Steadman, J.C. Rosas y J. Venegas. 2008. Co-evolution of the bean rust pathogen Uromyces appendiculatus with its wild, weedy and domesticated hosts (Phaseolus spp.) at a center of diversity. Annual Report of the Bean Improvement Cooperative 51: 2223.

Acevedo, M., J.C. Steadman y J.C. Rosas. 2012. Uromyces appendiculatus in Honduras: pathogen diversity and host resistance screening. Plant Disease (accepted for publication).

Araya, R., J.C. Hernández. 2007. Variedades de frijol de grano rojo obtenidas por fitomejoramiento participativo en Costa Rica 2000-2007. Programa de Investigación y Transferencia de Tecnología Agropecuaria de Frijol (PITTA-Frijol). Estación Experimental "Fabio Baudrit", Alajuela, Costa Rica. $20 \mathrm{p}$.

Beaver, J.S., G. Godoy, J.C. Rosas y J. R. Steadman. 2002. Estrategias para seleccionar frijol común con mayor resistencia a la mustia hilachosa. Agronomía Mesoamericana 13 (1): 67-72.

Beaver, J.S., J.C. Rosas, J. Myers, J. Acosta, J.D. Kelly, S. Nchimbi-Msolla, R. Misangu, S. Temple, E. ArnaudSantana y D. P. Coyne. 2003. Contributions of the Bean/Cowpea CRSP to cultivar and germplasm development in common bean. Field Crops Research 82: $87-102$.
Blair, M.W., J.S. Beaver y J.C. Rosas. 1994. Heritability of field resistance to bean golden mosaic virus in dry beans (Phaseolus vulgaris L.). In: F. Morales (ed.). Bean Golden Mosaic-Research Advances, ProFrijol/COSUDE/CIAT, Cali, Colombia. 160-170.

Frahm, M.A., J.C. Rosas, N. Mayek-Pérez, E. LópezSalinas, J.A. Acosta-Gallegos y J.D. Kelly. 2003. Resistencia a sequía terminal en frijol negro tropical. Agronomía Mesoamericana 14 (2):143-150.

Frahm, M.A., J.C. Rosas, N. Mayek-Pérez, E. LópezSalinas, J.A. Acosta-Gallegos y J.D. Kelly. 2004. Breeding beans for resistance to terminal drought in the lowland tropics. Euphytica 136:223-232.

González, N., F.H. Fewerda, M. Alameda, J.C. Rosas y J.S. Beaver. 2005. Identification of new sources of resistance to web blight of common bean. . Annual Report of the Bean Improvement Cooperative 48: 130131.

Graham, P.H. y J.C. Rosas. 1977. Growth and development of indeterminate bush and climbing cultivars of Phaseolus vulgaris L. inoculated with Rhizobium. Journal of Agricultural Science Cambridge 88:503508.

Graham, P.H. y J.C. Rosas. 1978a. Nodule development and Nitrogen Fixation in cultivars of Phaseolus vulgaris L. as influenced by plant density. Journal of Agricultural Science Cambridge 90: 19-29.

Graham, P.H. y J.C. Rosas. 1978b. Plant and nodule development and nitrogen fixation in climbing cultivars of Phaseolus vulgaris L. grown in monoculture or associated with Zea mays L. Journal of Agricultural Science Cambridge 90: 311-317.

Graham, P.H., J.C. Rosas, C. Estévez de Jensen, E. Peralta, B. Tlusty y J.A. Acosta-Gallegos. 2003. Addressing edaphic constraints to bean production: Bean/Cowpea CRSP perspective. Field Crops Research 82: 179-192.

Guachambala, M.S. y J.C. Rosas. 2010. Caracterización molecular de accesiones cultivadas y silvestres de frijol común de Honduras. Agronomía Mesoamericana 21(1):1-10.

Henry, A., J.C. Rosas, J.S. Beaver y J.P. Lynch. 2010. Multiple stress response and below ground competition in multilines of common bean (Phaseolus vulgaris L.). Field Crops Research 117:209-218.

Hernández, J.C., F.I. Elizondo. 2006. Estudio sobre la adopción de variedades mejoradas de frijol en las principales zonas productoras de frijol de la Región Brunca de Costa Rica. Agronomía Mesoamericana 17(3):357-367. 
Ho, M.D., J.C. Rosas, K.M. Brown y J.P. Lynch. 2005. Root architectural tradeoff for water and phosphorus adquisition. Functional Plant Biology 32:737-748.

Hocdé, H., J.C. Rosas y R. Araya. 2010. Co-Desarrollo de variedades entre agricultores, científicos $\mathrm{y}$ profesionales, biodiversidad y otras cosas. 2010. ISDA, Montpellier, Francia. 12 p.

Lynch, J.P. 2005. Root architecture and nutrient acquisition. In: Nutrient acquisition by plants: an ecological perspective. Ecological Studies 181:147-184.

Mather, D.L., R. Bernsten, J.C. Rosas, A. Viana y D. Escoto. 2003. The economic impact of diseaseresistant beans in Honduras. Agricultural Economics 29:343-352.

Meza, N., J.C. Rosas, J.P. Martín y J.M. Ortiz. 2012. Biodiversity of common bean (Phaseolus vulgaris L.) in Honduras, evidenced by morphological characterization. Genetic Resources and Crop Evolution (aceptado para publicación).

Porch, T., R. Bernsten, J.C. Rosas y M. Jahn. 2007. Climate change and the potential economic benefits of neattolerant bean varieties for farmers in Atlantida, Honduras. Journal of Agriculture of the University of Puerto Rico 91(3-4):133-148.

Reyes, B., M. Maredia y R. Bernsten. 2012. Variedades mejoradas de frijol generan beneficios económicos a agricultores. Evaluaciones de Impacto- Reporte de Investigación. Dry Grain Pulses CRSP, Julio 2012, No. 1, Michigan State University, 4 p.

Rodriguez, O. y J.C. Rosas. 2010. Selección de genotipos de frijol común por su resistencia al tizón bacteriano. Agronomía Mesoamericana 21(2):1-6.

Rosas, J.C. 1986a. Utilización del potencial de la fijación de nitrógeno en el frijol común in Central América. Ceiba 27(1): 105-115.

Rosas, J.C. 1986b. Improvement of the N2 fixation capacity in common beans. Ceiba 27(1):95-104.

Rosas, J.C. y F.A. Bliss. 1986. Improvement of the nitrogen fixation potential of common beans in Latin America. Ceiba 27(2): 245-260.

Rosas, J.C., J. Kipe-Nolt, R.A. Henson y F.A. Bliss. 1987. Estrategias de mejoramiento para incrementar la capacidad de fijación biológica de nitrógeno del frijol común en América Latina. Ceiba 28(1): 39-57.

Rosas, J.C., J.D. Erazo y J.R. Moncada. 1991. Tolerancia a la sequía en germoplasma de frijol común y frijol tepari. Ceiba 32 (2): 91-106.

Rosas, J.C., A.M. Andrews y J.A. Castro. 1996. Tolerancia del frijol común a la baja disponibilidad de fósforo en suelos de Honduras. Ceiba 37 (2): 1-6.
Rosas, J.C., O.I. Varela y J.S. Beaver. 1997. Registration of "Tío Canela 75" small red bean (race Mesoamerica). Crop Science 37:1391.

Rosas, J.C., A. Castro y E. Flores. 2000a. Mejoramiento genético de frijol rojo y negro Mesoamericano para Centro América y el Caribe. Agronomía Mesoamericana 11 (2): 37-46.

Rosas, J.C., A. Castro, J.S. Beaver, C.A. Pérez, A. Morales y R. Lépiz. 2000b. Mejoramiento genético para tolerancia a altas temperaturas y resistencia al mosaico dorado en frijol común. Agronomía Mesoamericana 11 (1):1-10.

Rosas, J.C. 2001. Aplicación de metodologías participativas para el mejoramiento genético del frijol común en Honduras. Agronomía Mesoamericana 12 (2): 219228.

Rosas, J.C., J.C. Hernández y R. Araya. 2003. Registration of 'Bribri' small red bean (race Mesoamerica). Crop Science 43 (1): 430-431.

Rosas, J.C., O. Gallardo y J. Jiménez. 2003. Mejoramiento genético del frijol común mediante enfoques participativos en Honduras. Agronomía Mesoamericana 14 (1):1-9.

Rosas J.C., J. S. Beaver, D. Escoto, C.A. Perez, A. Llano, J.C. Hernandez y R. Araya. 2004a. Registration of "Amadeus 77" small red bean. Crop Sci. 45:26562657.

Rosas, J.C., J.S. Beaver, S. Beebe y A. Viana. 2004b. Nomenclatura de las variedades de frijol liberadas en Centro América y el Caribe. Agronomía Mesoamericana 15 (2): 221-224.

Rosas, J.C., R. Araya e I. Ortega. 2009a. Variedades de frijol rojo obtenidas por itomejoramiento Participativo en Honduras y Nicaragua. Estación Experimenta "Fabio Baudrit", Alajuela, Costa Rica. 40 p.

Rosas, J.C., M.S. Guachambala y R.A. Ramos. 2009b. Guía ilustrada para la descripción de las características varietales del frijol común. Escuela Agrícola Panamericana, Zamorano, Honduras. 22 p.

Serracín, J., R.A. Young, J.C. Rosas y J. Cáceres. 1991. Damages caused by Xanthomonas campestris pv. phaseoli (common bacterial blight) and its effect on the yield of common bean. Journal of Agriculture of the University of Puerto Rico 75 (4):353-361.

Steadman, J.R., G. Godoy-Lutz, J.C. Rosas y J.S. Beaver. 2002. Uso de viveros móviles para determinar los patrones de virulencia de la roya del frijol. Agronomía Mesoamericana 13 (1): 37-39.

Recibido para publicación el 28 de noviembre de 2012. Aceptado para publicación el 15 de enero de 2013. 